Jerry P. Nolan

\title{
What's new in the management of cardiac arrest?
}

Received: 24 March 2013

Accepted: 26 March 2013

Published online: 24 April 2013

(C) Springer-Verlag Berlin Heidelberg and ESICM 2013

J. P. Nolan (®)

Anaesthesia and Intensive Care Medicine, Royal United Hospital, Combe Park, Bath BA1 3NG, UK

e-mail: jerry.nolan@nhs.net

Tel.: +44-1225-825056

Post-cardiac arrest patients account for a significant proportion of the occupied bed days in intensive care units (ICUs). This brief review will highlight some of the recent advances in cardiopulmonary resuscitation (CPR) and post-cardiac arrest care.

\section{Outcome}

Data published over the last 2 years indicate that survival rates following cardiac arrest are improving. In a study of out-of-hospital cardiac arrest (OHCA) from non-shockable rhythms in King County, Washington, 1-year survival rates over a 5-year period before implementation of new resuscitation guidelines in 2005 were compared with survival rates in the 5-year period post-implementation [1]. The survival rates increased from 2.7 to $4.9 \%$ [risk-adjusted odds ratio (OR) $1.85 ; 95 \%$ confidence interval (CI) 1.29-2.66]. Data from the American Heart Association's Get with the Guidelines-Resuscitation registry indicate that risk-adjusted rates of survival to discharge after in-hospital cardiac arrest (IHCA) have increased from $13.7 \%$ in 2000 to $22.3 \%$ in 2009 (adjusted rate ratio per year 1.04; $95 \%$ CI 1.03-1.06) [2].

\section{Prevention}

The two main interventions most likely to improve survival rates from IHCA are: (1) identification of the deteriorating patient and instigation of treatment to prevent cardiac arrest; (2) implementation of a do-not-attempt CPR decision in patients who will not benefit from CPR. Rapid response teams (RRTs) have replaced conventional resuscitation teams in many hospitals but, unfortunately, there is little evidence that they reduce cardiac arrest rates and mortality. After implementation of a RRT in an academic hospital in Riyadh, Saudi Arabia, non-ICU cardiac arrest rates decreased from 1.4 to 0.9 per 1,000 hospital admissions [relative risk (RR) 0.68 ; $95 \%$ CI $0.53-0.86$ ] and total hospital mortality decreased from 22.5 to 20.2 per 1,000 hospital admissions (RR 0.90; $95 \%$ CI 0.85-0.95) [3].

\section{CPR quality}

Quality of CPR is an important determinant of outcome. The North American Resuscitation Outcomes Consortium (ROC) investigators analysed 5-min periods of data downloaded from monitor-defibrillators in 3,098 OHCAs and concluded that a compression rate of $125 \mathrm{~min}^{-1}$ was associated with the maximum rate of return of spontaneous circulation (ROSC) [4]. Compression rates of $>125 \mathrm{~min}^{-1}$ were associated with a declining ROSC rate, probably because of the reduced compression depth that occurs at these higher compression rates $[4,5]$. In a prospective, cluster-randomised trial, the ROC investigators showed that monitor-defibrillators equipped to provide real-time audiovisual feedback improved the quality of CPR but did not influence ROSC or other clinical outcomes [6]. Following the implementation of scenario-based training and real-time audiovisual feedback, investigators from Arizona documented an increase 
in all-rhythm survival to hospital discharge from 8.7 to $13.9 \%$ (adjusted OR 2.72; $95 \%$ CI 1.15-6.41) [7].

\section{Advanced life support}

Tracheal intubation is generally perceived as the optimal method for managing the airway during cardiac arrest, but several large observational studies have challenged the value of tracheal intubation in OHCA. A Japanese registry of 649,654 consecutive adult OHCA patients showed that bag-mask ventilation was associated with improved neurological outcome when compared with tracheal intubation or use of a supraglottic airway (SGA) [8]. Multivariable logistic regression, adjusting for known confounders, showed worse neurological outcomes compared with bag-mask ventilation for both tracheal intubation (adjusted OR 0.41; $95 \%$ CI $0.37-0.45$ ) and SGAs (adjusted OR 0.38; $95 \%$ CI 0.36-0.40). A secondary analysis of the ROC PRIMED study comparing tracheal intubation $(n=8,487)$ with SGAs (laryngeal tube, Combitube or laryngeal mask airway; $n=1,968$ ) showed that successful tracheal intubation was associated with increased survival to hospital discharge (adjusted OR 1.40 ; $95 \%$ CI 1.04-1.89) when compared with SGA insertion [9]. Prospective randomised trials of airway management during cardiac arrest are urgently needed.

Although adrenaline is considered an essential drug for the treatment of cardiac arrest, placebo-controlled clinical trials are lacking. The only such trial to date showed an increased rate of ROSC with adrenaline but was not powered adequately to determine definitively if it improves survival to hospital discharge [10]. The largest study to date on the use of adrenaline in cardiac arrest involved 417,188 OHCAs in Japan [11]. In propensity-matched patients, use of adrenaline was associated with a 2.5 -fold higher ROSC rate (adjusted OR 2.51; $95 \%$ CI 2.24-2.80; $P<0.001$ ), but a 1-month survival rate approximately half of that achieved in those not given adrenaline (adjusted OR 0.54; $95 \%$ CI $0.43-0.68 ; P<0.001)$. Although this is a very large study and every effort has been made to eliminate bias by using multiple and comprehensive statistical analyses, hidden confounders may account for these findings. Alternatively, adrenaline could be genuinely harmful in cardiac arrest, perhaps by reducing microcirculatory flow in the brain despite increasing cerebral perfusion pressure. Appropriately powered placebo-controlled clinical trials of adrenaline in cardiac arrest are an urgent research priority.

\section{Post-resuscitation care}

In recent years, considerable progress has been made in the treatment of post-cardiac arrest patients. Primary percutaneous coronary intervention $(\mathrm{PCI})$ is the preferred method to re-establish myocardial perfusion after STelevation myocardial infarction (STEMI), and this is now accepted practice even in patients remaining comatose after OHCA. Increasing evidence supports widening the inclusion criteria for primary PCI after OHCA to include those patients with a likely cardiac cause of their cardiac arrest regardless of the electrocardiogram (ECG) findings, mainly because the ECG is difficult to interpret after ROSC. Approximately $25 \%$ of patients resuscitated after OHCA of likely primary cardiac cause, but who do not have ST elevation, are found to have an acute culprit lesion at early coronary angiography [12].

In addition to primary PCI, another intervention that is associated with improved outcome after OHCA is therapeutic hypothermia (TH). There is reasonably good evidence (two randomised controlled trials) supporting the use of $\mathrm{TH}$ after ventricular fibrillation/ventricular tachycardia OHCA, but evidence for its benefit after OHCA from non-shockable rhythms is restricted mainly to observational studies with substantial risk of bias [13]. In a prospective observational study involving 21 Finnish ICUs, 70/223 (31.4\%) of unconscious patients resuscitated from non-shockable rhythms were treated with TH but, even after adjustment for baseline differences, there was no difference in neurological outcome between those receiving or not receiving TH [14]. The Target Temperature Management (TTM) trial, which includes all-rhythm OHCAs, has recently finished recruiting patients and may provide some more reliable data on the impact of $\mathrm{TH}$ following non-shockable rhythm OHCA. The optimal method for cooling remains uncertain, but in a recent observational study, one-third of 594 post-cardiac arrest patients admitted to ICU and cooled externally failed to reach target temperature within $12 \mathrm{~h}$ [15]. Early angiography, high body weight and a long delay between collapse and start of cooling were among several independent risk factors for external cooling failure.

\section{Prognostication}

Modification of recovery from cerebral hypoxic-ischaemic injury by $\mathrm{TH}$, along with an increased use of sedatives and their reduced elimination during cooling, has made redundant previous guidelines on the prediction of poor outcome in the comatose post-cardiac arrest patient. The Glasgow Coma Scale motor score is unreliable $72 \mathrm{~h}$ after cardiac arrest [16] and even posthypoxic myoclonus, previously considered a reliable predictor of poor outcome, was followed by a good neurological outcome in $9 / 79$ patients $(12 \%)$ in one series [17]. The international resuscitation community is currently evaluating all available data with the aim of producing revised 
guidelines on prognostication in the near future. In the meantime, the Swedish Resuscitation Council has published recommendations on prognostication that, importantly, include delaying neurological evaluation until at least $72 \mathrm{~h}$ after rewarming when TH has been used [18].

Conflicts of interest JN is Editor-in-Chief of Resuscitation.

\section{References}

1. Kudenchuk PJ, Redshaw JD, Stubbs BA et al (2012) Impact of changes in resuscitation practice on survival and neurological outcome after out-ofhospital cardiac arrest resulting from nonshockable arrhythmias. Circulation 125:1787-1794

2. Girotra S, Nallamothu BK, Spertus JA, Li Y, Krumholz HM, Chan PS (2012) Trends in survival after in-hospital cardiac arrest. N Engl J Med 367:1912-1920

3. Al-Qahtani S, Al-Dorzi HM, Tamim HM et al (2013) Impact of an intensivist-led multidisciplinary extended rapid response team on hospital-wide cardiopulmonary arrests and mortality. Crit Care Med 41:506-517

4. Idris AH, Guffey D, Aufderheide TP et al (2012) Relationship between chest compression rates and outcomes from cardiac arrest. Circulation 125:3004-3012

5. Stiell IG, Brown SP, Christenson J et al (2012) What is the role of chest compression depth during out-ofhospital cardiac arrest resuscitation? Crit Care Med 40:1192-1198

6. Hostler D, Everson-Stewart S, Rea TD et al (2011) Effect of real-time feedback during cardiopulmonary resuscitation outside hospital: prospective, clusterrandomised trial. Br Med J 342:d512

7. Bobrow BJ, Vadeboncoeur TF, Stolz U et al (2013) The influence of scenariobased training and real-time audiovisual feedback on out-of-hospital cardiopulmonary resuscitation quality and survival from out-of-hospital cardiac arrest. Ann Emerg Med [Epub ahead of print]
8. Hasegawa K, Hiraide A, Chang Y, Brown DF (2013) Association of prehospital advanced airway management with neurologic outcome and survival in patients with out-ofhospital cardiac arrest. JAMA 309:257-266

9. Wang HE, Szydlo D, Stouffer JA et al (2012) Endotracheal intubation versus supraglottic airway insertion in out-ofhospital cardiac arrest. Resuscitation 83:1061-1066

10. Jacobs IG, Finn JC, Jelinek GA, Oxer HF, Thompson PL (2011) Effect of adrenaline on survival in out-of-hospital cardiac arrest: a randomised doubleblind placebo-controlled trial. Resuscitation 82:1138-1143

11. Hagihara A, Hasegawa M, Abe T, Nagata T, Wakata Y, Miyazaki S (2012) Prehospital epinephrine use and survival among patients with out-ofhospital cardiac arrest. JAMA 307:1161-1168

12. Dumas F, Cariou A, Manzo-Silberman $S$ et al (2010) Immediate percutaneous coronary intervention is associated with better survival after out-of-hospital cardiac arrest: insights from the PROCAT (Parisian Region Out of hospital Cardiac ArresT) registry. Circ Cardiovasc Interv 3:200-207

13. Kim YM, Yim HW, Jeong SH, Klem ML, Callaway CW (2012) Does therapeutic hypothermia benefit adult cardiac arrest patients presenting with non-shockable initial rhythms? A systematic review and meta-analysis of randomized and non-randomized studies. Resuscitation 83:188-196
14. Vaahersalo J, Hiltunen P, Tiainen M et al (2013) Therapeutic hypothermia after out-of-hospital cardiac arrest in Finnish intensive care units: the FINNRESUSCI study. Intensive Care Med [Epub ahead of print]

15. Ricome S, Dumas F, Mongardon N et al (2013) Predictors of external cooling failure after cardiac arrest. Intensive Care Med 39(4):620-628

16. Bouwes A, Binnekade JM, Kuiper MA et al (2012) Prognosis of coma after therapeutic hypothermia: a prospective cohort study. Ann Neurol 71:206-212

17. Bouwes A, van Poppelen D, Koelman JH et al (2012) Acute posthypoxic myoclonus after cardiopulmonary resuscitation. BMC Neurol 12:63

18. Cronberg T, Brizzi M, Liedholm LJ et al (2013) Neurological prognostication after cardiac arrestRecommendations from the Swedish Resuscitation Council. Resuscitation [Epub ahead of print] 